\section{Deutscher Röntgenkongress, 13. bis 16. Mai 2015 in Hamburg - MTRA-Programm}

Zum 5. und vorerst letzten Mal wird das Hamburger Congress Center ( $\mathrm{CCH})$ in diesem Jahr Gastgeber des größten radiologischen Fachkongresses Deutschlands sein. Wieder an seinem traditionellen Termin über Himmelfahrt werden erneut ca. 7000 Besucher erwartet. Ein umfangreiches, dreitägiges MTRA-Programm unter der wissenschaftlichen Leitung der VMTB startet am Donnerstagmorgen (14. Mai).

Der Kongress steht unter dem Motto „Technik für Menschen“. „Wie kein anderes Fach verkörpert die Radiologie die Symbiose ärztlicher Kunst und technischer Innovation“, so Kongresspräsident Prof. Dr. Gerhard Adam. „Wenige medizinische Disziplinen sind so technikgetrieben und technikaffin wie unser Fach. Daher wollen wir diesen Kongress auch in Kooperation mit technisch und naturwissenschaftlich orientierten Fachgesellschaften bestreiten, die unser Fach mit ihren Innovationen vorantreiben." Diesem Anspruch trägt auch das MTRA-Programm Rechnung. Themen zu Technologiefortschritt, Interventionelle Angiografie, Gefäßerkrankungen und zur Frage, wie konventionell wir eigentlich noch sind, finden sich im Programm und viele hochkarätige Vorträge werden auch die Nachbardisziplinen berühren.

Auch in diesem Jahr werden mit Sicherheit wieder die Klinikseminare ein Highlight sein, bei denen sich MTRA direkt am Universitätsklinikum Hamburg-Eppendorf praktisch fortbilden können: Die Intensiv-Kurse werden in den Bereichen Angiografie, Mammografie, MRT des Herzens, CT, Konstanzprüfung sowie Pädiatrisches Röntgen angeboten. Als neues Thema wird angeboten: „Gedanken sehen - Funktionelle Bildgebung in der Kernspintomographie“.

Weiterhin wird es für MTRA-Lehrkräfte wieder ein spezielles Lehrkräfte-Seminar geben sowie auch einige themenspezifische Workshops, die auf dem Kongressgelände stattfinden - z.B. die Workshops „Kommunikation und Gesprächsführung“, „MRT-Grundlagen“ oder „Artefakte in der Mammographie“. Da die Seminare sehr beliebt sind und pro Kurs nur wenige Plätze zur Verfügung stehen, empfiehlt sich eine frühzeitige Buchung.

Zum 1. Mal wird es in 2015 ein Programm extra für MTRA-Schüler geben. Die Veranstaltung wird im Saal Bucky stattfinden und ist kostenfrei für die Schüler. Am Freitagvormittag können sich die MTRA von Morgen über Themen wie Hygiene, Kontrastmittel, CT-Thorax, Neuroradiologie und vieles mehr informieren.

Herzlich einladen möchten wir alle VMTB-Mitglieder im Rahmen des Röntgenkongresses auch zu unserer diesjährigen ordentlichen Mitgliederversammlung am Donnerstag, 14. Mai 2015 um 13:30 Uhr, in dessen Rahmen ein neuer Vorstand gewählt wird sowie zu der gemeinsamen Eröffnungsveranstaltung von DRG und VMTB am selben Tag um 11:00 Uhr.

Weitere Informationen, das komplette Kongressprogramm und die Online-An-

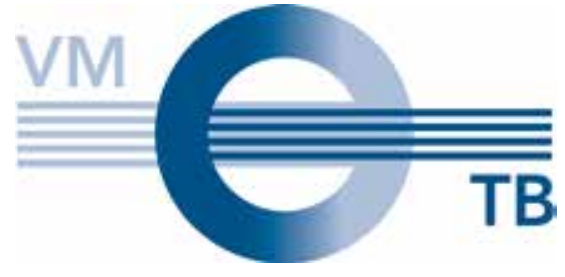

meldung erreichen Sie über www.roentgenkongress.de. Bis zum 6. April 2015 gibt es für VMTB- und RTaustria-Mitglieder über den Online-Frühbucherrabatt das Kongressticket für 70,00 €; Nichtmitglieder zahlen per Online-Buchung eine Kongressgebühr von 130,00€. Klinikseminare und MTRA-Workshops müssen wie gewohnt extra gebucht werden. Möchten Sie am ärztlichen Refresherkurs- und Workshop-Programm teilnehmen und hierfür eine Teilnahmebescheinigung erhalten, müssen Sie die Fortbildungspauschale in Höhe von 30,00 € zu Ihrem Kongressticket hinzu buchen (hiervon ausgenommen sind wie immer teilnehmerbegrenzte Kurse wie z.B. Intensiv- und Hands-OnWorkshops).

Wir freuen uns auf Sie in Hamburg!

Nina Keil, Pressestelle der DRG

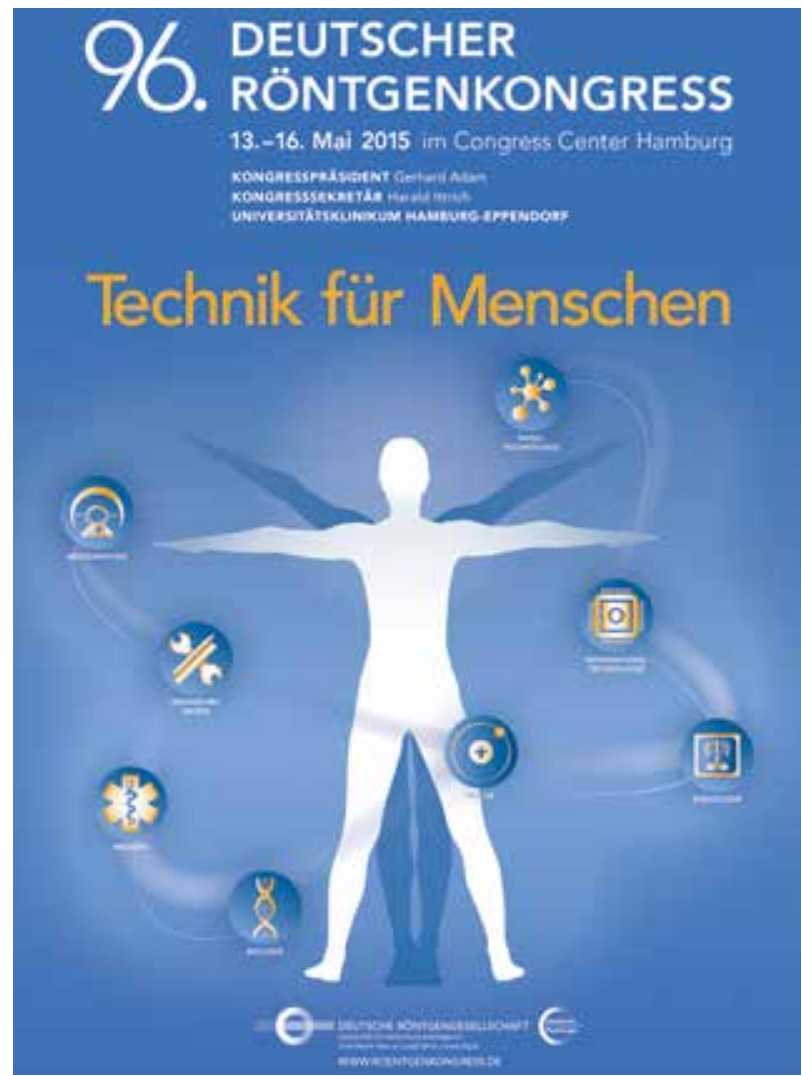

